\title{
A Note on Benford's Law for Second Order Linear Recurrences with Perodical Coefficients
}

\author{
P. SCHATtE and K. NAgASAKA
}

The solutions of second order linear recurrences with periodical coefficients are shown to obey Benford's law.

Key words: Benford's law, linear recurrences, uniform distribution of sequences

AMS subject classification: $11 \mathrm{KO6}$

1. Let $\left(u_{n}\right)$ be a sequence of real numbers which satisfy a second order $1 i-$ near recurrence

$$
u_{n+2}=a_{n+2} u_{n+1}+b_{n+2} u_{n}, n \geq 0,
$$

where $\left(a_{n}\right)$ and $\left(b_{n}\right)$ are given real sequences with a common period $r$, i.e., $a_{n+r}=a_{n}$ and $b_{n+r}=b_{n}$.

Linear recurrences of such type arise,e.g.in the theory of continued fractions.If $\omega$ is a quadraticirational,then the numerators and the denominators of the $n-t h$ convergent $p_{n} / q_{n}$ of the continued fraction of $w$ fulfil the relation (1) with $b_{n}=1$ and a periodic positive integer sequence (an) (cf..e.g..[4:Satz 1/p.S and Satz $28 / p .52$ ]). Linear recurrences with periodical coefficients are also treated.in [1:p.148-150].

The sequence $\left(u_{n}\right)$ is said to obey Benford's law if

$$
N^{-1} \mathfrak{z}\{n: 1 \leq n \leq N, 1 \leq \text { mantissa of } u<x\} \rightarrow \lg x \quad(N \rightarrow \infty)
$$

for $1<x<10$.Here $\lg x=\log _{10} x$ and $\sharp A$ is the number of the elements of $A$. More specially this means that

$$
N^{-1}\left\{\left\{n: 1 \leq n \leq N, u_{n} \text { has leading digit } k\right\} \rightarrow \lg (k+1)-\lg k,\right.
$$

for $k=1, \ldots, 9$ (cf.,e.g.,[9] for a survey on Benford's law). The sequence ( $u_{n}$ ) obeys Benford:s law if and only if the sequence $\left(\lg _{\mathrm{g}}\left|u_{n}\right|\right)$ is uniformly distributed mod 1 , put $\lg 0=1$ in this connection. For the uniform distribution mod 1 of sequences $c f ., e . g .,[6]$.

In case of $r=1$, i.e., $a_{n}=a$ and $b_{n}=b$, the solutions $\left(u_{n}\right)$ of $(1)$ obey Benford's law under weak suppositions. This case was extensively treated in the literature (cf.,e.g.,[3] for a survey and [8]).In the following we shall show that the solutions $\left(u_{n}\right)$ of (1) obey Benford's law also in the case $r>1$ by reducing this case to that of $r=1$. As a corollary we obtain 
that the numerators $\left(p_{n}\right)$ and the denominators $\left(q_{n}\right)$ of the $n$-th convergent $\rho_{n} / q_{n}$ of the continued fraction of a quadratic irrational $\omega$ obey Benford's law. Previous proofs of this fact can be found in [2], where a result from [5] is applied, and in [10]. Our result generalizes this assertion on continued fractions.

2. We put $z_{n, i}=u_{n r+i}$ for $0 \leq i \leq r-1$. Furthermore we introduce the shift operator $S$ and write $z_{n+1, i}=S z_{n, i}$. Then the linear recurrence (1) is equivalent to the system

$$
\begin{aligned}
& c_{1} z_{n, 0}+a_{2} z_{n, 1}-z_{n, 2}=0 \text {. } \\
& c_{2} z_{n, 0}+b_{3} z_{n, 1}+a_{3} z_{n, 2}-z_{n, 3}=0 \text {, } \\
& c_{r-2} z_{n, 0}+b_{r-1} z_{n, r-3}+a_{r-1} z_{n, r-2}-z_{n, r-1}=0 \text {, } \\
& c_{r-1} z_{n, 0}+b_{r} z_{n, r-2}+a_{r} z_{n, r-1}=0 \text {. } \\
& c_{r} z_{n, 0}-S z_{n, 1}+b_{1} z_{n, r-1}=0 \text {, }
\end{aligned}
$$

where $c_{1}=b_{2}, c_{2}=\ldots=c_{r-2}=0, c_{r-1}=-S, c_{r}=a_{1} S$. Now we multiply the $r-t h$ equation in this system by the signed minor of the element $c_{r}$ of the matrix of coefficients of this system. After summing up all the arising equations we arrive at

$$
\left|\begin{array}{ccccc}
b_{2} & a_{2} & -1 & & \\
& b_{3} & a_{3} & -1 & 0 \\
0 & \ddots & \ddots & \ddots & \\
-S & & b_{r-1} & a_{r-1}-1 \\
a_{1} S & -S & & b_{r} & a_{r} \\
& & & b_{1}
\end{array}\right| z_{n, 0}=0
$$

By expanding the determinant with respect to powers of $S$ we obtain finally

$$
z_{n+2,0}=D_{r} z_{n+1,0}+E_{r} z_{n, 0} .
$$

where

$$
E_{r}=(-1)^{r-1} b_{1} \cdots b_{r} \text { and } D_{r}=a_{1} A_{r}+b_{1} A_{r-1}+B_{r},
$$

with

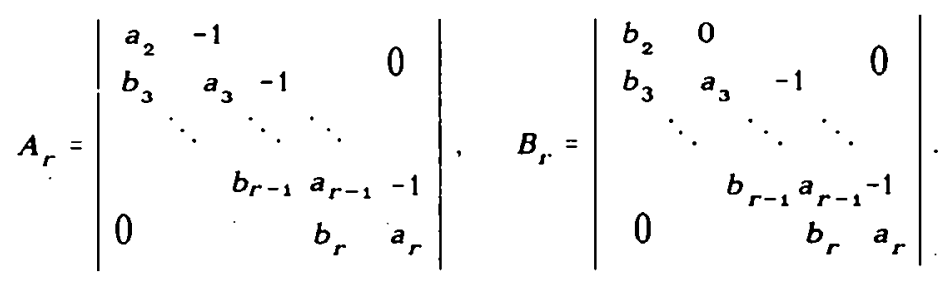


The determinants $A_{r}$ and $B_{r}$ are so-called continuants (cf.[7:p.8]).If we multiply the equations of (2) with the signed minors of the $i$-th column of the matrix of coefficients of $(2)$ and after that sum up,then we obtain

$$
z_{n+2, j}=D_{r} z_{n+1, j}+E_{r} z_{n, j}
$$

for $0 \leq j \leq r-1$ in generalization of (3).

3. Now we are in a position to formulate our main result.

Theorem 1: Let $\lambda_{1}, \lambda_{2}$ be real roots of the common characteristic equation

$$
\lambda^{2}=D_{r} \lambda+E_{r}
$$

of the linear recurrences (5); assume $\left|\lambda_{1}\right| \geq\left|\lambda_{2}\right|$.If $\lg \left|\lambda_{1}\right|$ is irrational and $u_{n} \neq 0$ for $n \geq n_{0}$, then the sequence $\left(u_{n}\right)$ obeys Benford's law.

Proof: Since the sequences $\left(z_{n, i}\right)$ fulfil (s), they all obey Benford's law as well-known (cf.,e.g.,[3]).But then the sequence $\left(u_{n}\right)$ obeys Benford's law, likewise

$$
\begin{aligned}
& \text { Remarka: 1.If we set } A_{0}=0, A_{1}=1, B_{0}=1, B_{1}=0, \text { then we get from (4) } \\
& A_{j}=a_{j} A_{i-1}+b_{i} A_{i-2}, B_{j}=a_{i} B_{i-1}+b_{i} B_{i-2}
\end{aligned}
$$

for $i \geq 2$. Thus we find that $A_{i}, B_{i} \geq 1, D_{i} \geq 3$ if $a_{j}, b_{j}$ are positive integers. 2.In order toderive yetanotherexpression for $D_{r}$ we rewrite ( 7 ) in the form

$$
\left(\begin{array}{ll}
A_{j} & A_{i-1} \\
B_{j} & B_{j-1}
\end{array}\right)=\left(\begin{array}{ll}
A_{i-1} & A_{i-2} \\
B_{i-1} & B_{i-2}
\end{array}\right)\left(\begin{array}{ll}
a_{i} & 1 \\
b_{j} & 0
\end{array}\right)
$$

(cf.[7:p.13]). Then we obtain

$$
D_{r}=\operatorname{trace}\left(\begin{array}{ll}
a_{1} & 1 \\
b_{1} & 0
\end{array}\right)\left(\begin{array}{ll}
A_{r} & A_{r-1} \\
B_{r} & B_{r-1}
\end{array}\right)=\operatorname{trace} \prod_{i=1}^{r}\left(\begin{array}{ll}
a_{1} & 1 \\
b_{i} & 0
\end{array}\right)
$$

(cf. [2:Equality (2.7)]). 3.The proof of Theorem 1 works also if some $b_{1}=0$ and therefore $E_{r}=0$.In this case $D_{r} \neq 0$ must hold since otherwise $u_{n}=0$ for $n=2 r$. 4. The line of reasoning can easily be generalized to linear recurrences of higher order.

4. From the main result we conclude the following

Corollary : Let $p_{n} / q_{n}$ be the $n-t h$ convergent of the continued fraction of a quadratic irrational $w$. Then the sequences $\left(p_{n}\right)$ and $\left(q_{n}\right)$ obey Benford's law.

Proof: The sequences $\left(\rho_{n}\right)$ and $\left(q_{n}\right)$ satisfy recurrences of type (1) with $b_{n}=1$ and positive integers $a_{n}$. The case $r=1$ being treated in [ 3 : Theorem 3.1] we can restrict to that of $r>1$. But then $D_{r} \geq 3$ according to Remark 1. Therefore the roots 


$$
\lambda_{1}=\left(D_{r}+\sqrt{D_{r}^{2}-4(-1)^{r}}\right) / 2, \lambda_{2}=\left(D_{r}-\sqrt{D_{r}-4(-1)^{r}}\right) / 2
$$

of $(6)$ are real and irrational.If $\lambda_{2}=10^{p / q}$ with integers $\rho$ and $q$, then $\lambda_{1}^{q}$ $=10^{P}$ must be an integer which is an obvious contradiction, apply the binomial theorem

5. Finally we shortly treat the case of complex conjugate roots of $(6)$,i.e., $D_{r}^{2}-4 E_{r}<0$. Let $\beta=\sqrt{E_{r}}, \cos 2 \pi \gamma=D_{r} / 2 \beta$.

Theorem 2 : If $1, \lg \beta$, and $\gamma$ are linearly independent over the rationals and $u_{n} \neq 0$ for $n \geq n_{0}$, then the sequence $\left(u_{n}\right)$ obeys Benford's law.

Proof : Apply Corollary 2 in [8] or Theorem 2.1 in [3]

\section{REFERENCES}

[1] Berg,L.: Lineare Glelchungssystememit Bandstruktur.Berlin:Dt.Verlag Wiss. 1986 .

[2] Jager, H.,and P.Liardet:Distributions arithmetiques des denominetors de convergents de fractions continues.Indag.Math. 91 (1988),181-197.

[3] Kanemitsu.S., Nagasaka,K.,Rauzy,G.,and J.-S.Shiue: On Benford's law The first digit problem.Lect. Notes Math. 1299 (1988),158-169.

[4] Khintchine,A.: KettenbruchelLeipzig: B.G.Teubner Verlagsges. 1956.

[5] Kiss,P.: On second order recurrences and continued fractions. Bull. Malaysian Math Soc.(2) 5(1982),33-41.

[6] Kuipers, L.,and H.Niederreiter: Uniform distribution of sequences. New York: J.Wiley. \& Sons 1974.

[7] Perron.O.: Die Lehrevon den Kettenbruchen,Bd.I.Stuttgart:B.G.Teubner Verlagsges. 1954.

[8] Schatte,P.: Onthe uniform distribution of certain sequences and Benford's law. Math. Nachr. 136 (1988),271-273.

[9] Schatte,P.:On mantissadistributions incomputing and Benford's law. J.Inf.Process.Cybern. EIK 24(1988),443-455.

[10] Schate, P.: On Benford's law for continued fractions.Math.Nachr. 148 $(1990)$ ( to appear).

Received 21.06.1989

Author's address:

Doz.Dr.Peter Schatte

Sektion Mathematik

der Bergakademie Freiberg

Bernhard-von-Cotta-Str. 2

D (Ost) - 9200 Freiberg

Dr.Kenji Nagasaka

The University of the Alr

2-11, Wakaba

Chiba City

260 Japan 